\title{
Long noncoding RNA LINC02582 acts downstream of miR-200c to promote radioresistance through CHK1 in breast cancer cells
}

\author{
Baiyao Wang ${ }^{1}$, Jieling Zheng ${ }^{2}$, Rong Li', Yunhong Tian², Jie Lin², Yingying Liang ${ }^{2}$, Quanquan Sun ${ }^{3}$, Anan Xu', \\ Ronghui Zheng', Mengzhong Liu'2, Aimin $\mathrm{Ji}^{2}$, Junguo Bu${ }^{4}$ and Yawei Yuan ${ }^{1,2}$
}

\begin{abstract}
Radiotherapy is essential to treat breast cancer and microRNA (miRNA) miR-200c is considered as a radiosensitizer of breast cancer. However, the molecular mechanisms by which miR-200c regulates radiosensitivity remain largely unknown. In the present study, we showed that induction of miR-200c led to widespread alteration in long noncoding RNA (IncRNA) expression in breast cancer cells. We identified IncRNA LINC02582 as a target of miR-200c. Inhibition of LINC02582 expression increased radiosensitvity, while overexpression of LINC02582 promoted radioresistance. Mechanistically, LINC02582 interacts with deubiquitinating enzyme ubiquitin specific peptidase 7 (USP7) to deubiquitinate and stabilize checkpoint kinase 1 (CHK1), a critical effector kinase in DNA damage response, thus promoting radioresistance. Furthermore, we detected an inverse correlation between the expression of miR-200c vs. LINC02582 and CHK1 in breast cancer samples. These findings identified LINC02582 as a downstream target of miR200c linking miR-200c to CHK1, in which miR-200c increases radiosensitivity by downregulation of CHK1.
\end{abstract}

\section{Introduction}

Radiation therapy plays an important role in the multidisciplinary management of breast cancer. It not only provides local control and reduces tumor local relapse but also increases patients' long-term survival and decreases their mortality ${ }^{1,2}$. However, radioresistance is a major cause of failure of breast cancer radiation therapy ${ }^{3}$. Therefore, the molecular mechanisms involved in breast cancer radioresistance should be investigated.

MicroRNAs (miRNAs) regulate a wide range of biological processes and dysregulation of miRNAs contributes to

Correspondence: Junguo Bu (flkd007@smu.edu.cn) or Yawei Yuan (yuanyawei@gzhmu.edu.cn)

'Department of Radiation Oncology, Nanfang Hospital, Southern Medical University, Guangzhou, Guangdong Province, People's Republic of China ${ }^{2}$ Department of Radiation Oncology, Affiliated Cancer Hospital \& Institute of Guangzhou Medical University, Guangzhou, Guangdong Province, People's Republic of China

Full list of author information is available at the end of the article. These authors contributed equally: Baiyao Wang, Jieling Zheng, Rong Li Edited by A. Stephanou cancer ${ }^{4,5}$. MiR-200c is a member of the miR-200 superfamily and is involved in stemness, epithelial-mesenchymal transition, and chemoresistance in various cancer cells ${ }^{6-8}$. Moreover, miR-200c has been identified as a critical regulator of radiosensitivity in several types of cancer, including breast cancer. MiR-200c sensitizes breast cancer cells to radiation by targeting TBK1 and EGFR ${ }^{9,10}$. Our previous study also showed that miR-200c sensitizes breast cancer cells to radiation by inhibiting radiation-induced autophagy ${ }^{11}$. Importantly, therapeutic delivery of miR-200c markedly increased radiosensitivity in several types of cancer $^{12,13}$. Thus, miR-200c acts as a tumor radiosensitizer and might represent an attractive target to increase the efficacy of radiation therapy for breast cancer. However, the molecular mechanisms by which miR-200c regulates radiosensitivity require further investigation.

Long noncoding RNAs (lncRNAs) are a large class of non-protein-coding transcripts with a length $>200$ bases ${ }^{14}$. LncRNAs have important roles in various biological processes in cancer, including proliferation, invasion,

\section{(c) The Author(s) 2019}

(c) (i) Open Access This article is licensed under a Creative Commons Attribution 4.0 International License, which permits use, sharing, adaptation, distribution and reproduction c. in any medium or format, as long as you give appropriate credit to the original author(s) and the source, provide a link to the Creative Commons license, and indicate if changes were made. The images or other third party material in this article are included in the article's Creative Commons license, unless indicated otherwise in a credit line to the material. If material is not included in the article's Creative Commons license and your intended use is not permitted by statutory regulation or exceeds the permitted use, you will need to obtain permission directly from the copyright holder. To view a copy of this license, visit http://creativecommons.org/licenses/by/4.0/. 
and metastasis ${ }^{15,16}$. For instance, IncRNA HOTAIR interacts with polycomb repressive complex 2 to reprogram chromatin, thus promoting breast cancer invasion and metastasis ${ }^{17}$. Furthermore, lncRNA NKILA is a negative regulator of NF- $\mathrm{kB}$ signaling, inhibiting NF- $\mathrm{kB}-$ mediated metastasis in breast cancer. Low NKILA expression predicts poor clinical outcome in patients with breast cancer ${ }^{18}$. In addition, lncRNA EPIC1 is an oncogenic lncRNA that interacts with MYC to promote cellcycle progression in breast cancer. High expression of EPIC1 is associated with poor prognosis in patients with breast cancer ${ }^{19}$. Therefore, lncRNAs represent a wide range of potential targets for cancer treatment. However, the role of lncRNAs in radiosensitivity is unclear.

Studies have shown that miRNAs interact with lncRNAs to regulate lncRNA levels ${ }^{20}$. For example, miR-211 inhibits lncRNA loc285194 expression ${ }^{21}$; miR-17-3p directly targets lncRNA GCASPC and decreases its half-life ${ }^{22}$; and miR-193b suppresses lncRNA MIR31HG expression ${ }^{23,24}$. Given that miR-200c can increase the radiosensitivity of breast cancer cells and that the contribution of miR-200c to IncRNA expression has not been assessed, we hypothesized that lncRNAs might be critical downstream targets of miR-200c in regulating radiosensitivity.

In the present study, we used microarray analysis to delineate the alterations in IncRNA expression induced by miR-200c. We identified lncRNA LINC02582 as a downstream target of miR-200c. LINC02582 is required for radioresistance in breast cancer cells. Mechanistically, LINC02582 interacts with deubiquitinating enzyme ubiquitin specific peptidase 7 (USP7) to deubiquitinate and stabilize checkpoint kinase 1 (CHK1), thereby promoting radioresistance.

\section{Results}

Overexpression of miR-200c enhances the radiosensitivity of breast cancer cells

To confirm that miR-200c sensitizes breast cancer cells to radiation, we first determined the miR-200c expression level in several breast cancer cell lines. Consistent with a previous report ${ }^{25}$, miR-200c is commonly expressed in breast cancer cells (Fig. 1a). Compared with miR-200c high-expression cell lines (MCF-7, BT474), miR-200c low-expression cell lines (MDA-MB-231, BT549, SKBR3, T47D) showed higher clonogenic survival after irradiation (Fig. 1b). These data indicated a positive correlation between miR-200c expression and radiosensitivity. MDAMB-231 and BT549 cells were transduced with lentivirus expressing miR-200c (Fig. 1c). Overexpression of miR200c reduced the survival fraction of MDA-MB-231 and BT549 cells subjected to irradiation (Fig. 1d). Conversely, inhibition of miR-200c increased the survival fraction of MCF-7 and BT474 cells after irradiation (Fig. 1e, f). Irradiation caused double-stranded DNA breaks (DSBs) with formation of $\gamma-\mathrm{H} 2 \mathrm{AX}$ foci, which indicated delayed repair and correlated with radiosensitivity. Indeed, miR200c overexpression led to persistence of $\gamma-\mathrm{H} 2 \mathrm{AX}$ foci in MDA-MB-231 cells at $24 \mathrm{~h}$ after irradiation (Fig. 1g, h). Analysis of $\gamma$-H2AX protein levels showed that miR-200c overexpression significantly increased $\gamma-\mathrm{H} 2 \mathrm{AX}$ levels after irradiation (Fig. 1i). These results confirmed that overexpression of miR-200c suppresses DNA repair and sensitizes breast cancer cells to radiation.

\section{Identification of miR-200c-associated IncRNAs}

To identify miR-200c-associated lncRNAs, differential expression of IncRNAs between MDA-MB-231 cells stably overexpressing miR-200c and control cells was assessed using microarray analysis. Hierarchical clustering showed variations in lncRNA expression between miR-200c-overexpressing cells and control cells (Fig. 2a). We found that $424 \operatorname{lncRNAs}$ were upregulated and 521 lncRNAs were downregulated (fold change $>2, P<0.05$ ) in miR-200c-overexpressing cells. To validate the results of microarray analysis, we randomly selected 10 lncRNAs showing $>10$-fold change and determined their expression using qRTPCR. Our data were consistent with the microarray results (Supplementary Fig. 1a). Previous studies demonstrated that interactions between miRNAs and lncRNAs represent an important gene regulation pathway $^{20}$. Among the downregulated IncRNAs, we predicted 58 potential lncRNA targets for miR-200c using the TargetScan and miRbase prediction algorithm (Supplementary Table 1). Taken together, the results suggested that overexpression of miR-200c lead to widespread alterations in IncRNA expression.

\section{LINC02582 is a direct target of miR-200c}

To investigate lncRNAs regulated by miR-200c, we selected five most differentially expressed candidate lncRNAs from among the potential targets of miR-200c and determined their expression in breast cancer cells (Supplementary Fig. 1b). Interestingly, among the five candidate lncRNAs, we found that LINC02582 (NCBI number NR_038340) expression was higher in the radioresistant breast cancer lines, including MDA-MB-231, BT549, SKBR3, T47D with low miR-200c levels, than in the radiosensitive cell lines, including MCF-7, BT474 with high miR-200c levels (Fig. 2b). This result revealed a negative correlation between LINC02582 expression and miR-200c expression and implied that LINC02582 was a possible target of miR-200c. Therefore, LINC02582 was chosen for further investigation. LINC02582 is located on human chromosome 18q22.3. The sequence of LINC02582 was identified by $5^{\prime}$ and $3^{\prime}$ RACE. Analysis of LINC02582 sequences by Open Reading Frame Finder from NCBI failed to predict an encoded protein, 
$\mathbf{a}$

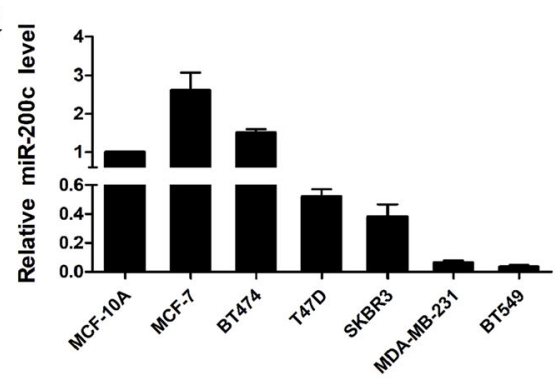

c

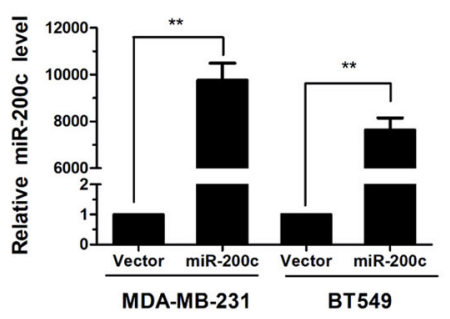

$\mathbf{e}$

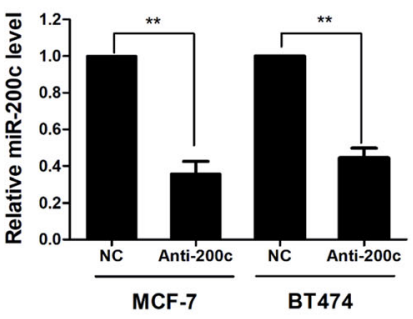

g

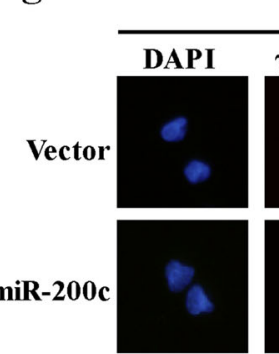
0Gy
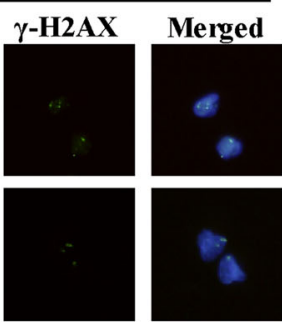

MDA-MB-231

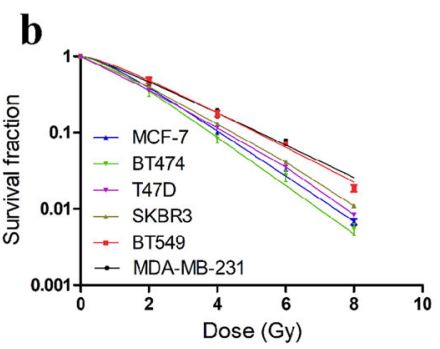

d
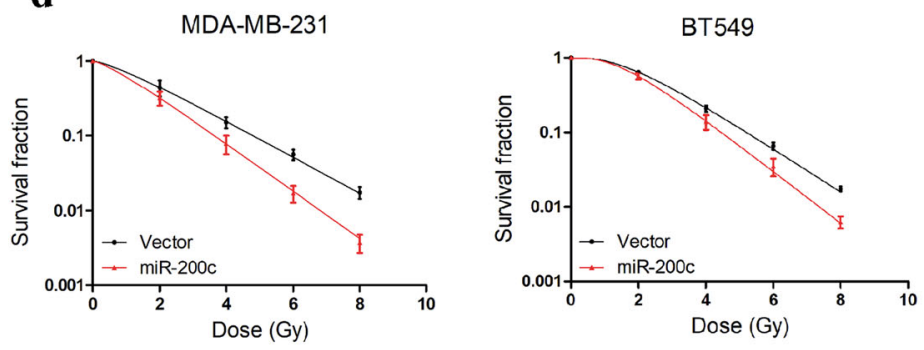

f
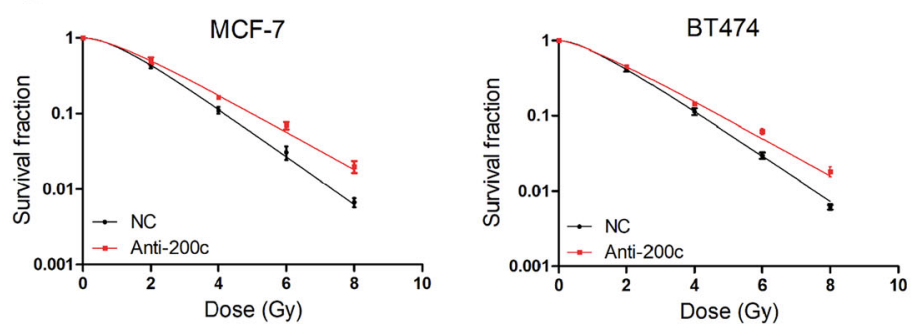

h

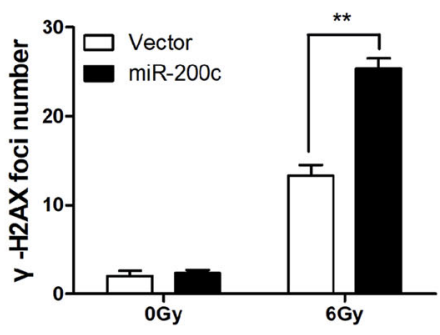

MDA-MB-231

i

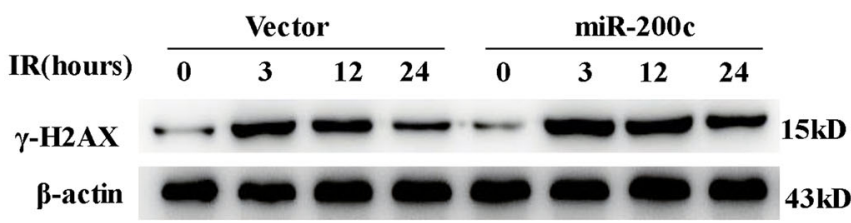

MDA-MB-231

Fig. 1 MiR-200c overexpression increases the radiosensitivity of breast cancer cells. a Relative expression of miR-200c in breast cancer cells and MCF-10A cells were detected using qRT-PCR. b Clonogenic survival assays of MDA-MB-231, BT549, SKBR3, T47D, BT474, and MCF-7 cells. c Relative expression of miR-200c in MDA-MB-231 and BT549 cells transduced with lentivirus encoding miR-200c or the empty vector. $\mathbf{d}$ Clonogenic survival assays of MDA-MB-231 and BT549 cells transduced with miR-200c. e Relative expression of miR-200C in MCF-7 and BT474 cells after transfection the miR-200C inhibitor. $\mathbf{f}$ Clonogenic survival assays of MCF-7 and BT474 cells transfected with the miR-200c inhibitor. $\mathbf{g}, \mathbf{h} \gamma$-H2AX foci formation analyzed by immunofluorescence in MDA-MB-231 cells transduced with miR-200c or empty vector, $24 \mathrm{~h}$ after $6 \mathrm{~Gy}$ IR. i Western blotting analysis of $\gamma^{-}$ H2AX expression in MDA-MB-231 cells transduced with miR-200c, at the indicated time points after 6 Gy IR. Data are presented as means \pm SD, $n=3$, ${ }^{*} P<0.05,{ }^{*} P<0.01$ 

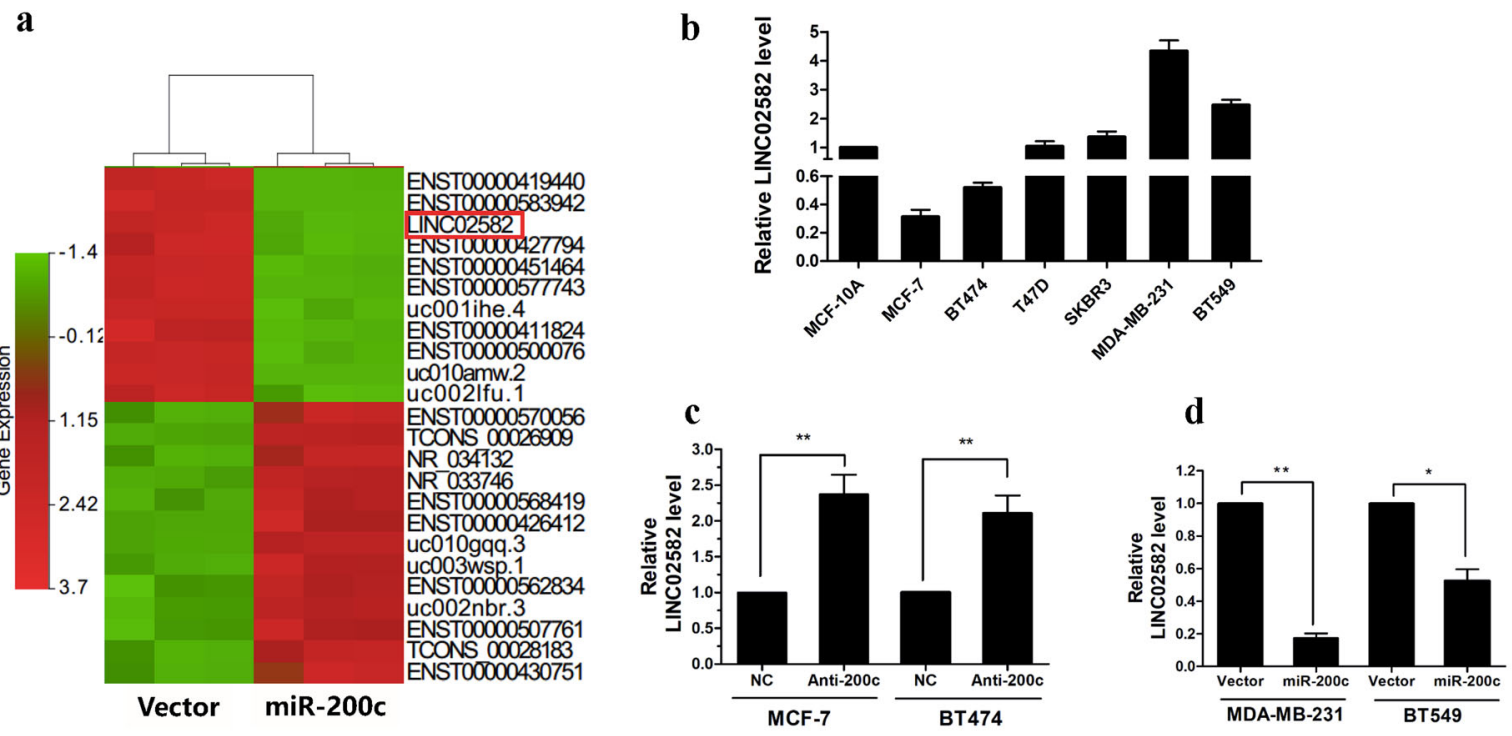

e
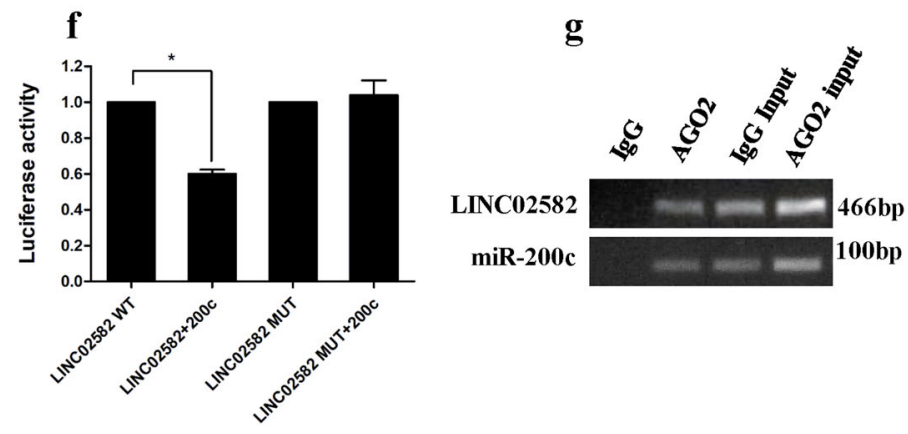

LINC02582 WT: 5'-TTAACTCCAAAT-CAACAGTA-TTT-3'

miR-200c: 3'-AGGUAGUAAUGGGGCGUCAUAAU-5' LINC02582 MUT:5'-TTAACTCCAAAGCAACUCAU-ATT-3' miR-200c: 3'-AGGUAGUAAUGGGCCGUCAUAAU-5'
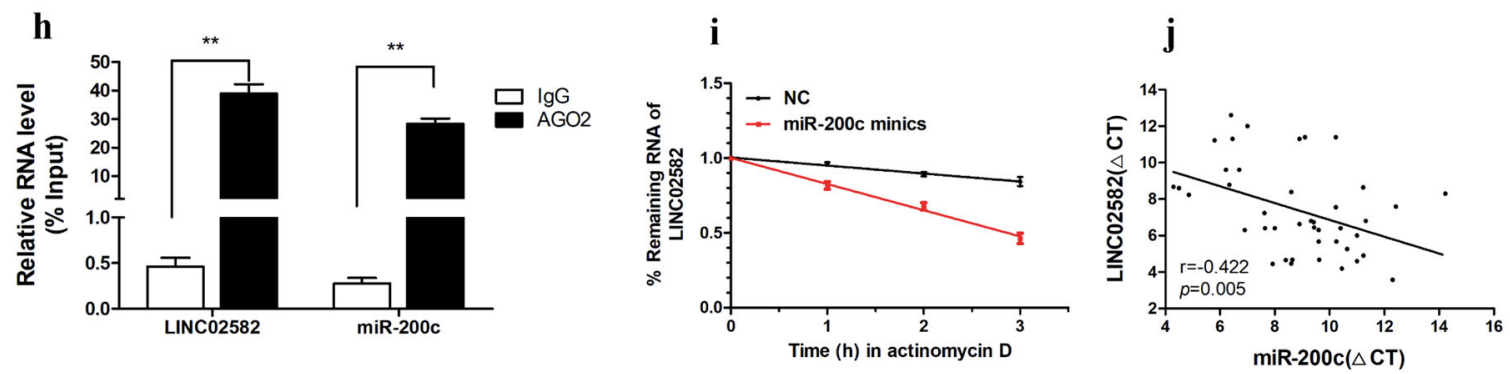

Fig. 2 LINC02582 is a target of miR-200c. a Representative heat map of the IncRNAs that were most differentially expressed between miR-200coverexpressing cells and control cells. b Relative expression of LINC02582 in breast cancer cells and MCF-10A cells. c Relative expression of LINC02582 in MCF-7 and BT474 cells after transfection with the miR-200c inhibitor. d Relative expression of LINC02582 in MDA-MB-231 and BT549 cells after transduction with miR-200c. e Target site of miR-200c in the LINC02582 sequence, as predicted by TargetScan and miRBase (upper panel). $\mathbf{f}$ Relative luciferase activity of MDA-MB-231 cells after co-transfection with wild type or mutant LINC02582 vectors and an miR-200c mimic. $\mathbf{g}$ Anti-AGO2 RIP to investigated AGO2 binding to miR-200c and LINC02582; IgG was used as a negative control. $\mathbf{h}$ The levels of miR-200c and LINC02582 were detected by qRT-PCR and presented as fold enrichment of AGO2 relative to input. $\mathbf{i}$ The half-life of LINC02582 was shortened by miR-200c overexpression. MDA-MB-231 cells were transfected with miR-200c mimics, after $48 \mathrm{~h}$, a time course for RNA stability was started by adding the actinomycin D. The cells were harvested at the indicated time points. The expression levels of LINC02582 were detected by qRT-PCR. $\mathbf{j}$ Expression levels of LINC02582 and miR-200c were negatively correlated in 42 breast cancer samples, as measured by qRT-PCR. The $\triangle C T$ values were subjected to Pearson correlation analysis. Data are presented as means $\pm S D, n=3,{ }^{*} P<0.05,{ }^{* *} P<0.01$

suggesting that LINC02582 has no protein-coding potential (Supplementary Table 2).

To investigate whether miR-200c regulates the expression of LINC02582, we suppressed miR-200c expression in MCF-7 and BT474 cells, which led to a significant increase in LINC02582 expression (Fig. 2c). Conversely, ectopic expression of miR-200c resulted in a significant reduction in LINC02582 in MDA-MB-231 and BT549 cells (Fig. 2d). However, we found no significant difference in miR-200c expression between LINC02582 
knockdown or overexpression cells (Supplementary Fig. 2a). Subsequently, TargetScan and miRBase predicted the existence of interactions between miR-200c and LINC02582 (Fig. 2e). To confirm the binding between LINC02582 and miR-200c, we performed dual-luciferase assays, which showed that miR-200c decreased the luciferase activity of the wild-type LINC02582 vector, but not that of the mutant LINC02582 vector (Fig. 2f). MiRNAs are known to bind their targets and cause translational repression or RNA degradation in an AGO2-dependent manner. To test whether miR-200c regulates LINC02582 in such a manner, we conducted an RNA immunoprecipitation (RIP) experiment in MDA-MB-231 cells using anti-AGO2 antibodies. The results showed that LINC02582 and miR-200c were enriched in AGO2 immunoprecipitates relative to IgG immunoprecipitates, which confirmed direct binding between miR-200c and LINC02582 (Fig. 2g, h). Moreover, ectopic expression of miR-200c shortened the half-life of LINC02582 (Fig. 2i). Finally, qRT-PCR revealed a negative correlation between miR-200c and LINC02582 expression in 42-paired samples of breast cancer tissue (Fig. 2j). These results indicated that LINC02582 is a target of miR-200c.

\section{LINC02582 inhibition increases radiosensitivity of breast cancer cells}

To evaluate the biological functions of LINCO2582, we inhibited its expression in MDA-MB-231 and BT549 cells (Fig. 3a), which had no effect on the viability of the breast cancer cells (Supplementary Fig. 2b). Interestingly, inhibition of LINCO2582 expression reduced the surviving fraction of MDA-MB-231 and BT549 cells after irradiation (Fig. 3b). Conversely, overexpression of LINC02582 increased the surviving fraction of MCF-7 and BT474 cells after irradiation (Fig. 3c, d). Furthermore, LINC02582 silencing led to persistence of $\gamma-\mathrm{H} 2 \mathrm{AX}$ foci in MDA-MB231 cells and BT549 cells at $24 \mathrm{~h}$ after irradiation (Fig. 3e, f, Supplementary Fig. 2c, d). LINC02582 silencing also markedly increased $\gamma$-H2AX expression after irradiation. (Fig. 3g, Supplementary Fig. 2e). These results indicated that silencing of LINCO2582 enhanced the radiosensitivity of breast cancer cells. To further investigate the effects of LINC02582 in vivo, MDA-MB-231 cells with LINC02582 knockdown were used to create a xenograft model (Supplementary Fig. 2f). When the tumor volume reached $150 \mathrm{~mm}^{3}$, local tumor irradiation was performed using a 2Gy fractionated dose every other day for 10 days (Fig. 3i). Knockdown of LINCO2582 had no effect on the growth of non-irradiated tumors, but caused tumor growth inhibition after irradiation (Fig. 3j, k). Taken together, these results demonstrated that silencing of LINCO2582 enhanced the radiosensitivity of breast cancer cells both in vitro and in vivo.

\section{LINC02582 interacts with USP7}

Studies have shown that some lncRNAs perform their functions by interacting with specific proteins ${ }^{26,27}$. To evaluate whether LINCO2582 acts via this mechanism, we performed RNA pull-down assays to identify proteins that interact with LINCO2582, followed by mass spectrometry analysis of the specific protein band for LINC02582 (Supplementary Table 3). Among the proteins identified by mass spectrometry, USP7 was also detected by western blotting (Fig. 4a, b). An RIP experiment using anti-USP7 antibodies in extracts from MDA-MB-231 cells showed enrichment of LINC02582 (but not GAPDH mRNA) using the USP7 antibodies vs. a nonspecific IgG control antibody (Fig. 4c, d). Moreover, deletion mapping analysis identified a 422-789 nt region of LINC02582 that was required for the interaction between LINC02582 and USP7 (Fig. 4e). Taken together, these results suggested that a specific interaction occurs between USP7 and LINC02582.

\section{LINC02582 functions through its interaction with USP7}

USP7 is a deubiquitinating enzyme involved in regulating the stability of many proteins ${ }^{28-30}$. USP7 promotes radioresistance of breast cancer cells by controlling CHK1 protein stability via direct deubiquitination ${ }^{31}$. Consistently, inhibition of USP7 reduced CHK1 protein levels and decreased radiosensitivity, while overexpression of USP7 elevated CHK1 protein levels and promoted radioresistance (Fig. 4f, g, Supplementary Fig. 3a). CHK1 is a critical effector kinase in the DNA damage response, which facilitates DNA damage repair and promotes radioresistance of breast cancer cells ${ }^{32}$. As expected, knockdown of CHK1 sensitized MCF-7 cells to radiation, whereas overexpression of $\mathrm{CHK} 1$ resulted in radioresistance of MDA-MB-231 cells (Fig. 4h, i, Supplementary Fig. 3b). We hypothesized that the interaction between LINC02582 and USP7 would lead to deubiquitination and stabilization of CHK1, which in turn would promote radioresistance.

To test this hypothesis, we first showed that knockdown of LINCO2582 reduced the CHK1 level, while ectopic expression of LINC02582 increased the CHK1 level (Fig. 5a). Importantly, LINC02582 inhibition or ectopic expression did not affect the CHK1 mRNA level (Supplementary Fig. 3c, d). To assess the effect of LINCO2582 on CHK1 stability, we treated MCF-7 cells with cycloheximide (CHX) to inhibit protein synthesis and detected the level of remaining CHK1 using western blotting. The abundance of CHK1 in LINC02582-overexpressing cells remained relatively higher than that in the control cells (Fig. 5b), suggesting that LINC02582 overexpression stabilized CHK1. Moreover, treatment of MDA-MB-231 cells silenced for LINC02582 with the proteasome 


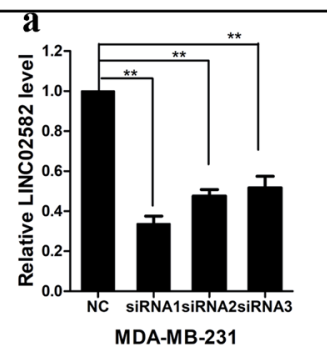

c

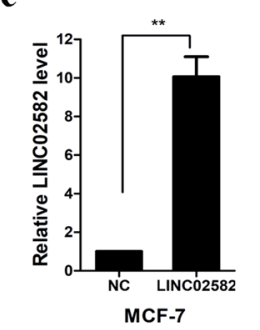

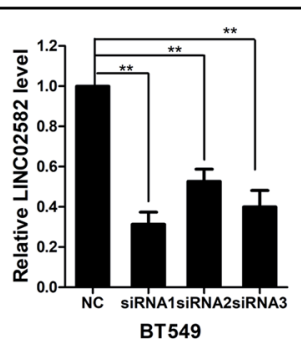

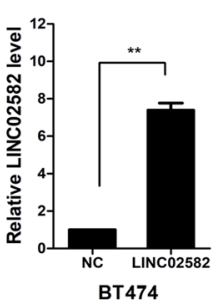

b

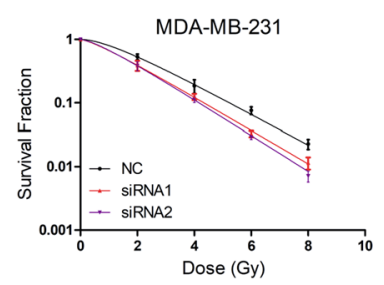

d

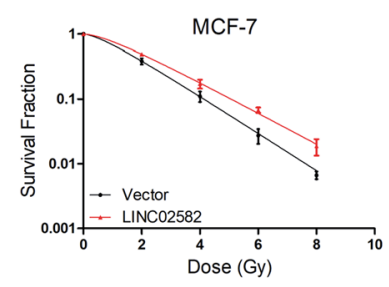

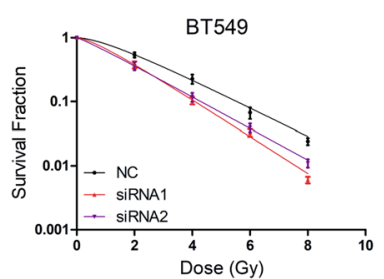

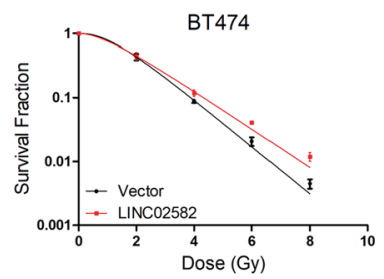

e

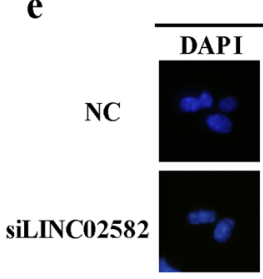

$0 \mathrm{~Gy}$
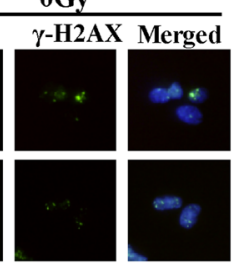

MDA-MB-231

g

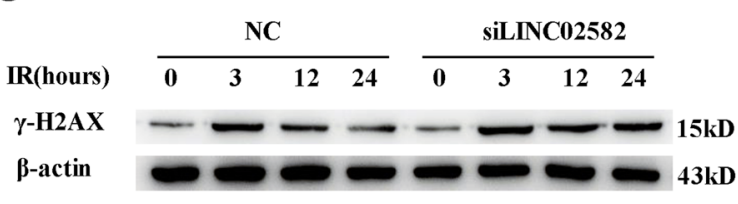

MDA-MB-231

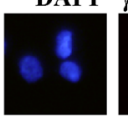

\begin{tabular}{|c|}
\hline $\begin{array}{c}6 \\
3 \\
3\end{array}$ \\
\hline 4 \\
4 \\
4
\end{tabular}

f

$\frac{6 \mathrm{~Gy}}{\gamma-\mathrm{H} 2 \mathrm{AX}}$ Merged
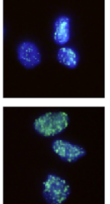

29.

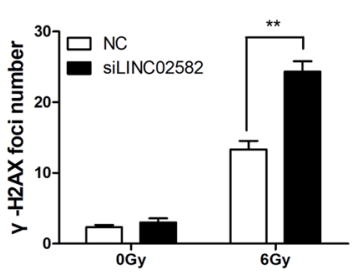

MDA-MB-231

h

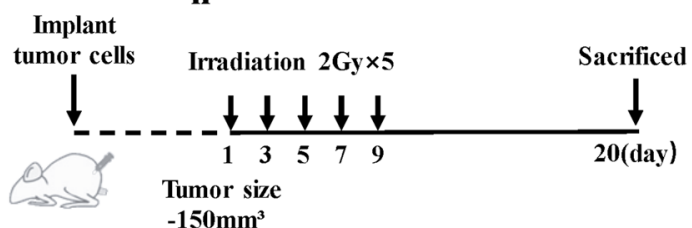

$-150 \mathrm{~mm}^{3}$
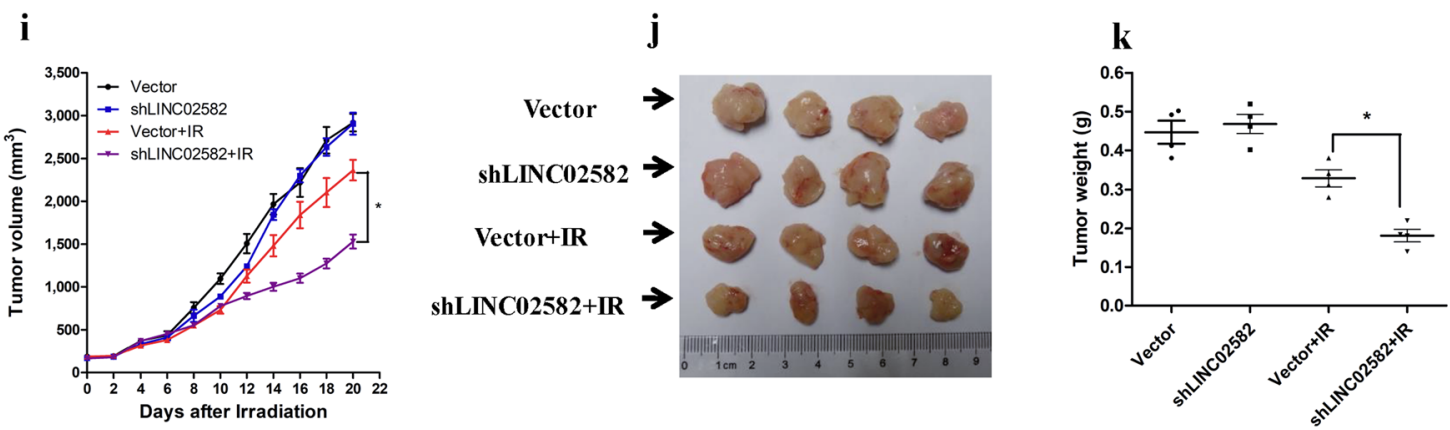

Fig. 3 LINC02582 promotes radioresistance of breast cancer cells. a Expression of LINC02582 in MDA-MB-231 and BT549 cells transfected with three different siRNAs corresponding to LINC02582. d Clonogenic survival assays of MDA-MB-231 and BT549 cells transfected with two different LINC02582 siRNAs. c Expression of LINC02582 in MCF-7 and BT474 cells transduced with lentivirus encoding the LINC02582 sequence. d Clonogenic survival assay of MCF-7 and BT474 cells transduced with LINC02582. e, f Formation of $\mathrm{Y}$-H2AX foci at $24 \mathrm{~h}$ after 6 Gy IR, analyzed by

immunofluorescence in MDA-MB-231 cells transfected with LINC02582 siRNA1. Data are presented as means \pm SD, $n=3,{ }^{*} P<0.05,{ }^{* *} P<0.01$.

g Western blotting analysis of $\mathrm{Y}$-H2AX expression in MDA-MB-231 cells transfected with LINC02582 siRNA1, at the indicated time points after 6 Gy IR. $\mathbf{h}$ Schemes for the establishment and treatment of the breast cancer orthotopic mouse model. $\mathbf{i}$ Tumor size in mice bearing control MDA-MB-231 xenografts or LINC02582 shRNA-transduced MDA-MB-231 xenografts. Data points show the mean tumor volume $\left(\mathrm{mm}^{3}\right)$ of each group $(n=4)$; bars, SE. ${ }^{*} P<0.05$. $\mathbf{j}$ Photographs of tumors developing in normal control (Vector), shLINC02582, Vector + irradiation (Vector + IR), and shLINC02582 + IR mice are presented. $\mathbf{k}$ Weight of tumors from the mouse model; tumor weights in the shLINC02582 + IR mice were lower than those from the Vector + IR mice. Data points show the mean tumor weight $(\mathbf{g})$ of each group $(n=4)$; bars, SE. ${ }^{*} P<0.05$ 


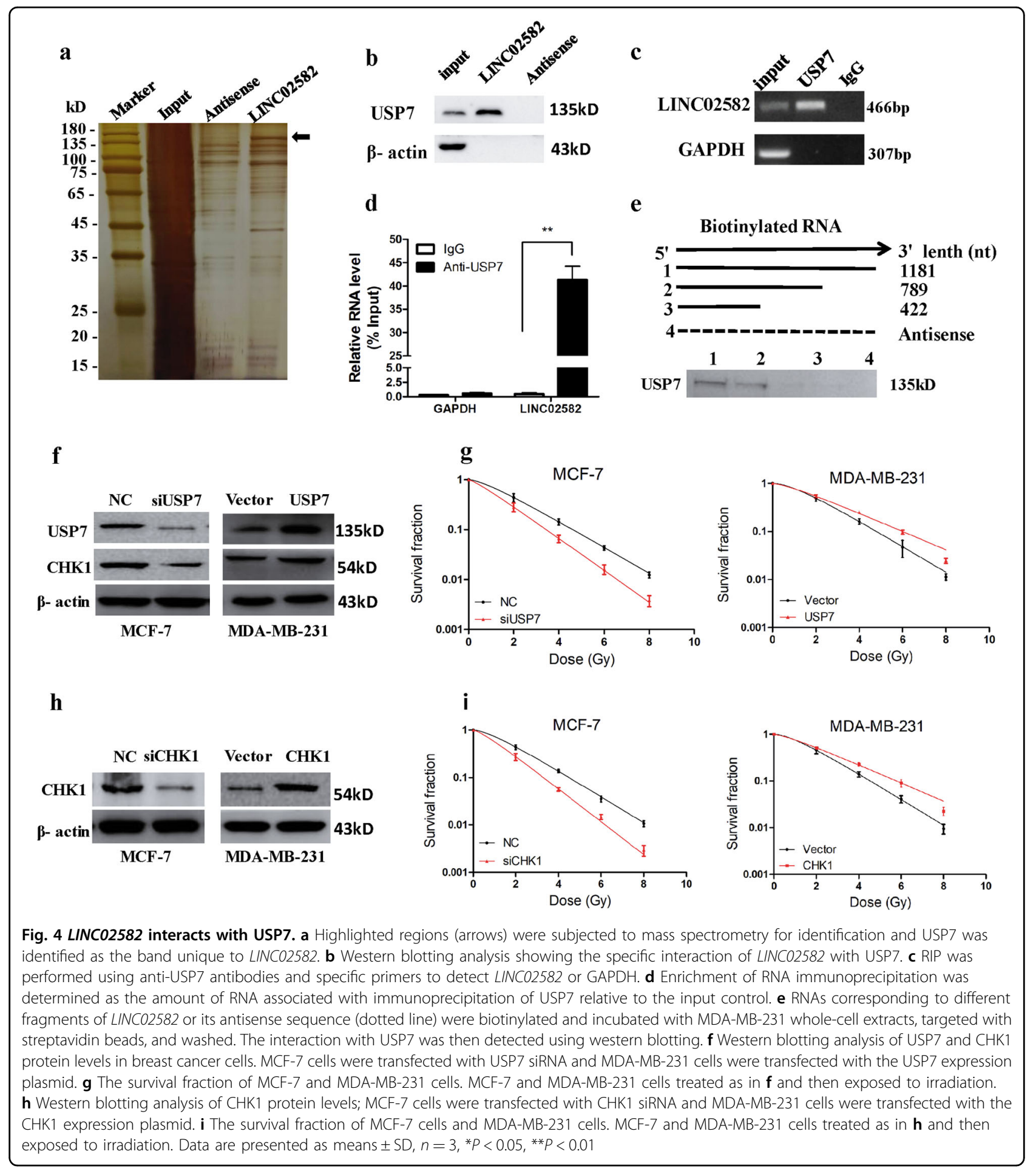

inhibitor MG132 resulted in increased endogenous CHK1 protein levels compared with those in control cells (Fig. 5c), suggesting that the ubiquitin-proteasome pathway plays a critical role in the LINC02582-mediated upregulation of CHK1.
Indeed, inhibition of LINC02582 in MDA-MB-231 cells promoted endogenous ubiquitination of CHK1 (Fig. 5d). On the contrary, overexpression of LINC02582 in MCF-7 cells induced endogenous deubiquitination of CHK1 (Fig. 5e); however, inhibition of USP7 blocked the 


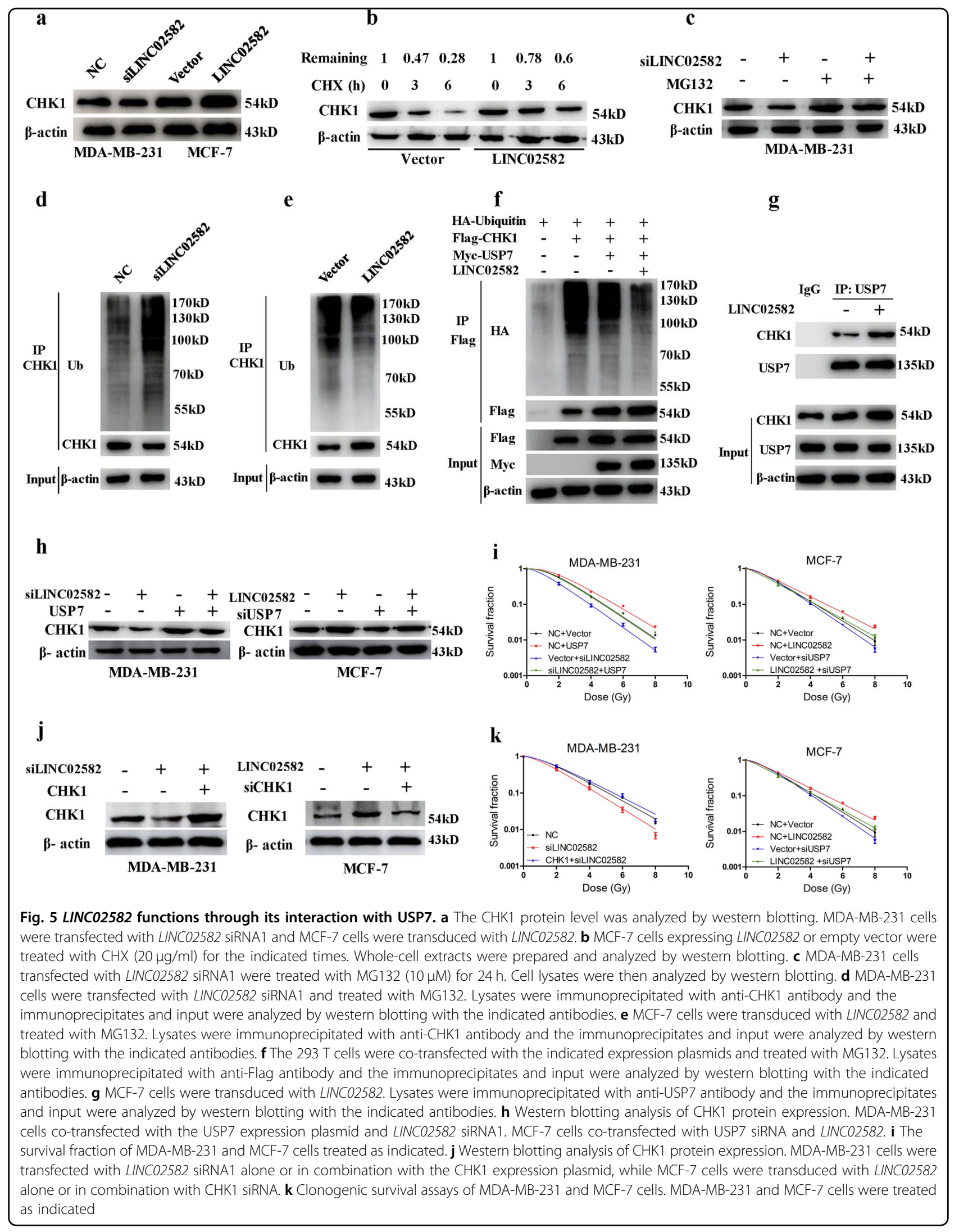


deubiquitination inducted by LINC02582 overexpression (Supplementary Fig. 4a). Furthermore, to detect the ubiquitination of CHK1, the $293 \mathrm{~T}$ cells were co-transfected with HA-tagged Ubiquitin, Flag-tagged CHK1, Myctagged USP7, and LINC02582 expression plasmids. Consistently, overexpression of USP7 significantly induced deubiquitination of CHK1 in 293 T cells; importantly, overexpression of LINC02582 promoted USP7-induced deubiquitination of CHK1 (Fig. 5f). Finally, we found that overexpression of LINCO2582 could enhance the interaction between USP7 and CHK1 (Fig. 5g). Collectively, these results suggested that LINCO2582 enhances the interaction between USP7 and CHK1, thereby increasing the deubiquitination of CHK1.

Importantly, we observed that the reduction in CHK1 caused by inhibition of LINC02582 was reversed by USP7 overexpression, while the upregulation of CHK1 caused by LINC02582 ectopic expression could be reversed by USP7 knockdown (Fig. 5h). The induction of radiosensitivity by LINC02582 inhibition was blocked by USP7 overexpression; however, the radioresistance induced by ectopic expression of LINC02582 could be blocked by USP7 knockdown (Fig. 5i). Similarly, ectopic expression of CHK1 in LINC02582-knockdown cells rescued the radioresistance, whereas knockdown of $C H K 1$ reversed the LINC02582-induced radioresistance (Fig. 5j, k). These results confirmed that the effect of LINCO2582 on radioresistance was at least partly dependent on USP7 and CHK1.

\section{MiR-200c enhances radiosensitivity by downregulation of CHK1}

We sought to determine whether miR-200c could regulate radiosensitivity through $\mathrm{CHK} 1$. We found that suppression of miR-200c elevated CHK1 protein levels while ectopic expression of miR-200c decreased CHK1 levels. Knockdown of LINC02582 blocked the CHK1 upregulation induced by miR-200c suppression, and LINC02582 ectopic expression rescued the CHK1 downregulation induced by miR-200c ectopic expression (Fig. 6a). Importantly, knockdown of LINC02582 prevented the induction of radioresistance caused by inhibition of miR-200c, while upregulation of LINCO2582 reversed the radiosensitivity caused by overexpression of miR-200c (Fig. 6b). Furthermore, knockdown of USP7 blocked the upregulation of CHK1 induced by miR-200c suppression and USP7 ectopic expression rescued the downregulation of CHK1 induced by miR-200c ectopic expression (Fig. 6c). Interestingly, knockdown of USP7 prevented the induction of radioresistance caused by inhibition of miR-200c, while upregulation of USP7 reversed the radiosensitivity caused by overexpression of miR-200c (Fig. 6d). Moreover, knockdown of CHK1 prevented the induction of radioresistance caused by inhibition of miR-200c, while upregulation of CHK1 reversed the radiosensitivity caused by overexpression of miR-200c (Fig. 6e, f). Taken together, these findings demonstrated that miR-200c enhances the radiosensitivity of breast cancer cell by downregulating the miR-200c/ LINC02582/USP7/CHK1 signaling axis, thereby playing a critical role in regulating the radiosensitivity of breast cancer cells.

\section{MiR-200C and LINC02582 expression correlate with CHK1 protein expression in breast cancer}

To confirm the association between miR-200c, LINC02582, and CHK1 in breast cancer specimens, we performed ISH or IHC to examine the expression of these molecules in 136 breast cancer tissue samples from patients with breast cancer who had received radiotherapy (Fig. 6g). Decreased expression of miR-200c was associated with elevated CHK1 expression. However, a positive correlation between the expression of LINC02582 and CHK1 was observed in these breast cancer specimens. Briefly, $72.6 \%$ (53/73) of the tumors with low miR-200c expression exhibited high CHK1 expression, and $77.4 \%$ (55/71) of the tumors with high LINC02582 expression showed high CHK1 expression (Fig. 6h). Neither clinical or pathological characteristics were correlated with the expression of LINC02582 (Supplementary Table 4). Kaplan-Meier analysis showed that patients with low miR-200c expression had significantly worse recurrencefree survival and patients with high LINC02582 expression exhibited worse recurrence-free survival (Fig. 6i, j).

\section{Discussion}

The results of the present study indicated that ectopic expression of miR-200c led to widespread alterations in lncRNA expression in breast cancer cells. We identified LINC02582 as a downstream target of miR-200c. LINC02582 is required for radioresistance in breast cancer cells. Mechanistically, LINC02582 interacts with USP7 to deubiquitinate and stabilize CHK1, thus promoting radioresistance. These findings indicated that the miR200c/LINC02582/USP7/CHK1 signaling axis play a critical role in regulating the radiosensitivity of breast cancer cells (Fig. 6k).

There is accumulating evidence that aberrant expression of lncRNAs is associated with several types of cancer, and lncRNAs play a key role in a wide range of cancer biological processes ${ }^{33,34}$. However, the effect of IncRNAs in radiosensitivity is largely unknown. It has been reported that miR-200c sensitizes breast cancer cells to radiation by targeting Ubiquilin 1 and TBK1 (refs. ${ }^{9,11}$ ). However, whether miR-200c can enhance radiosensitivity through lncRNAs has not been previously investigated. In the present study, we found that overexpression of miR$200 \mathrm{c}$ led to widespread alteration in lncRNA expression. 

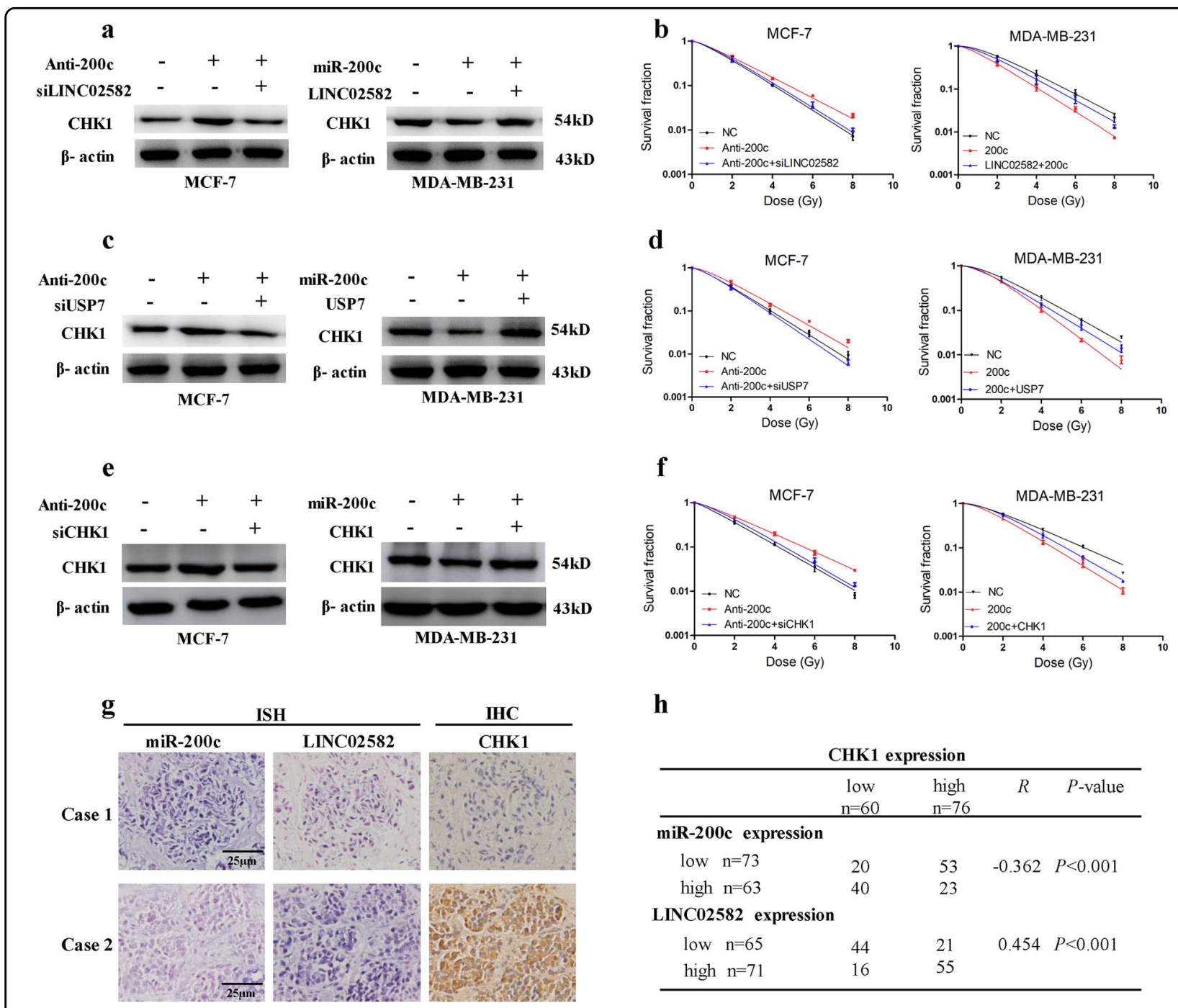

$\mathbf{h}$

\begin{tabular}{lllll}
\multicolumn{5}{c}{ CHK1 expression } \\
\hline & $\begin{array}{l}\text { low } \\
\mathrm{n}=60\end{array}$ & $\begin{array}{l}\text { high } \\
\mathrm{n}=76\end{array}$ & $R$ & $P$-value \\
\hline miR-200c expression & & & & \\
low $\mathrm{n}=73$ & 20 & 53 & -0.362 & $P<0.001$ \\
high $\mathrm{n}=63$ & 40 & 23 & & \\
LINC02582 expression & & & & \\
low $\mathrm{n}=65$ & 44 & 21 & 0.454 & $P<0.001$ \\
high $\mathrm{n}=71$ & 16 & 55 & & \\
\hline
\end{tabular}
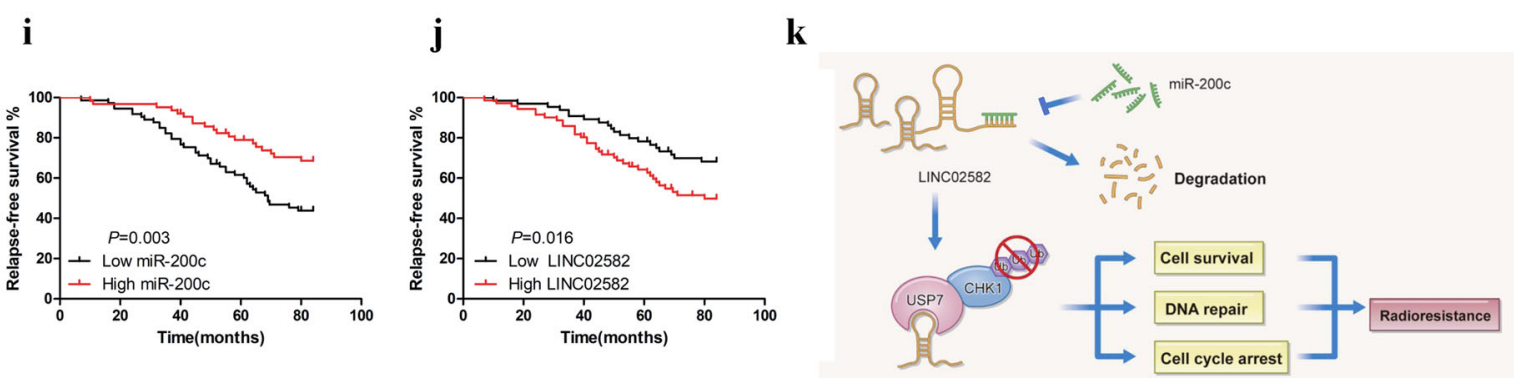

Fig. 6 MiR-200c increases radiosensitivity through downregulation of CHK1. a Western blotting analysis of CHK1 protein levels. MCF-7 cells were co-transfected with an miR-200c inhibitor and LINC02582 siRNA1; MDA-MB-231 cells were co-transfected with an miR-200c mimic and LINC02582. $\mathbf{b}$ The survival fraction of MDA-MB-231 and MCF-7 cells. MDA-MB-231 and MCF-7 cells treated as in a and then exposed to irradiation. c The CHK1 protein level was detected by western blotting. MCF-7 cells were co-transfected with an miR-200c inhibitor or with USP7 siRNA; MDAMB-231 cells were co-transfected with an miR-200c mimic or with the USP7 expression plasmid. $\mathbf{d}$ The survival fraction of MDA-MB-231 and MCF-7 cells. MDA-MB-231 and MCF-7 cells treated as in c and then exposed to irradiation. e Western blotting analysis of CHK1 protein expression. MCF-7 cells were co-transfected with an miR-200c inhibitor or with CHK1 siRNA; MDA-MB-231 cells were co-transfected with an miR-200c mimic and the CHK1 expression plasmid. f Clonogenic survival assays of MCF-7 and MDA-MB-231 cells. MCF-7 and MDA-MB-231 cells were treated as in e and then exposed to irradiation. g MiR-200c and LINC02582 expression were detected via in situ hybridization. CHK1 expression was detected via immunohistochemistry. $\mathbf{h}$ Correlations between the expression of miR-200c, LINC02582, and CHK1 in 136 clinical breast cancer specimens. The $p$ values were obtained using the $x^{2}$ test. i Kaplan-Meier curves showing recurrence-free survival of 136 breast cancer patients who were high or low for miR-200c expression. $\mathbf{j}$ Kaplan-Meier curves showing recurrence-free survival of 136 breast cancer patients who were high or low for LINC02582 expression. $\mathbf{k}$ Working model of the regulation of radiosensitivity by miR-200c 
Among the differentially expressed lncRNAs, we identified lncRNA LINCO2582 as a downstream target of miR200c and LINCO2582 was required for radioresistance in breast cancer cell. Breast cancer is a heterogeneous group of tumors with different estrogen receptor (ER)/progesterone receptor (PR)/human epidermal growth factor receptor 2 (HER2) statuses. In the present study, we found that knockdown of LINC02582 in MDA-MB-231 and BT549 cells (both ER-/PR-/HER2-) significantly increased radiosensitivity. In contrast, ectopic expression of LINC02582 in MCF-7 cell (ER+/PR+/HER2-) and BT474 cells (ER+/PR +/HER2+) contributed to raodioresistance. These results indicated that the effects of LINC02582 on raodiosensitivity was independent of the specific subtype of breast cancer cells. Our studies revealed that lncRNAs are an important component of the miR-200c regulatory network, and highlighted the important regulatory relationships between miRNAs and lncRNAs. However, the role of other lncRNAs showing differential expression in our microarray analysis requires further investigation in a future study.

Many studies have shown that some lncRNAs function by interacting with specific proteins ${ }^{35}$. In this study, we performed RNA pull-down accompanied by mass spectrometric assays to identify LINCO2582-interacting proteins. Notably, USP7 was confirmed as a specific binding protein for LINC02582. USP7 is a deubiquitinating enzyme that is involved in regulating the stability of CHK1. CHK1 is a critical effector kinases in the DNA damage response that facilitates DNA damage repair and promotes radioresistance of breast cancer cells ${ }^{36}$. Several studies reported that CUL1- and CUL4-containing E3 ubiquitin ligase complexes target CHK1 for polyubiquitylation and degradation ${ }^{37}$. A recent study indicated that USP7 increases CHK1 levels by direct deubiquitination, thereby promoting radioresistance in breast can$\mathrm{cer}^{31}$. Therefore, we reasoned that LINC02582 might interact with USP7 to deubiquitinate CHK1, thus promoting radioresistance. Consistently, the ubiquitination of CHK1 was markedly decreased in cells overexpressing LINC02582; however, USP7 inhibition blocked the deubiquitination of CHK1 induced by LINC02582 overexpression. Overexpression of LINC02582 enhances the interaction between USP7 and CHK1. Moreover, either knockdown of USP7 or CHK1 blocked the radioresistance induced by LINCO2582 overexpression. LINCO2582 promotes radioresistance through, at least partly, USP7 and CHK1. Our results suggested that LINC02582 enhances the interaction between USP7 and CHK1, thereby increasing the deubiquitination of $\mathrm{CHK} 1$ and promoting radioresistance.

MiR-200c inhibits DNA repair after radiation and acts as a radiosensitizer in breast cancer cells ${ }^{9}$. CHK1 facilitates DNA damage repair and promotes radioresistance ${ }^{31,38}$.
Importantly, CHK1 is overexpressed in breast cancer ${ }^{39}$. CHK1 inhibition is a promising radiosensitizing strategy for breast cancer. Here, we found that expression of miR200c reduced the CHK1 protein level, while inhibition of miR-200c upregulated the CHK1 protein level. Ectopic expression of CHK1 reversed the radiosensitivity induced by miR-200c. These results indicated that the effect of miR-200c on radiosensitivity in breast cancer cells is at least partly mediated by CHK 1 . We also identified LINCO2582 as a link between miR-200c, CHK1, and radioresistance of breast cancer cells. To the best of our knowledge, this is the first report to identify CHK1 as a downstream factor regulated by miR-200c. The miR-200c/ LINC02582/USP7/CHK1 signaling axis plays important role in regulating the radiosensitivity of breast cancer cells.

We identified a negative correlation between miR-200c and CHK1 expression, as well as a positive correlation between LINC02582 and CHK1 expression, in clinical breast cancer tissue samples. Importantly, in patients with breast cancer who had received radiotherapy, tumors showing low miR-200c or high LINC02582 expression had much worse recurrence-free survival. However, whether miR-200c and LINC02582 expression influences the overall survival of patients with breast cancer requires further investigation.

In summary, we demonstrated that LINC02582 is a downstream target of miR-200c. LINC02582 is required for radioresistance in breast cancer cells. Mechanistically, LINC02582 interacts with USP7 to deubiquitinate and stabilize CHK1, thus promoting radioresistance. Our results suggested that the miR-200c/LINC02582/USP7/ CHK1 signaling axis is a potential target to improve the response of breast cancer to radiation therapy.

\section{Material and methods \\ Patient and tumor specimens}

Paraffin-embedded samples of carcinomas were obtained from 136 patients with breast cancer in three hospitals, including Nanfang Hospital of Southern Medical University (Guangzhou 510515, China), Zhujiang Hospital of Southern Medical University (Guangzhou 510282, China), and Zhejiang Cancer Hospital (Hangzhou 310022, China), from January 2005 and January 2008. All these patients had received radiotherapy after surgery. Fresh tumor tissues were obtained from 42 breast cancer cases in Nanfang Hospital of Southern Medical University. All samples were collected after the patients signed informed consent according to the internal review and ethics boards of these hospitals. The diagnosis of breast cancer was confirmed histopathologically.

\section{Cell culture}

MCF-10A cells were obtained from the American Tissue Culture Collection (ATCC, Manassas, VA, USA). 
MDA-MB-231, MCF-7, BT549, SKBR3, T47D, and BT474 cells were obtained from the Cell Bank of Type Culture Collection (Chinese Academy of Sciences, Shanghai, China). MCF-10A cells were cultured in Dulbecco's modified Eagle's medium (DMEM)/F12 (1:1) medium supplemented with 5\% horse serum (Hyclone, Logan, UT, USA), $100 \mathrm{ng} / \mathrm{ml}$ cholera toxin (Sigma-Aldrich, St. Louis, $\mathrm{MO}, \mathrm{USA}$ ), $20 \mathrm{ng} / \mathrm{ml}$ epidermal growth factor (EGF; Peprotech, Rocky Hill, NJ, USA), $0.5 \mu \mathrm{g} / \mathrm{ml}$ hydrocortisone (Sigma-Aldrich), and $10 \mu \mathrm{g} / \mathrm{ml}$ insulin (SigmaAldrich). MCF-7, SKBR3 and T47D cells were maintained in DMEM containing 10\% fetal bovine serum (FBS; Gibco, Melbourne, Australia). All other cell lines were cultured in Roswell Park Memorial Institute (RPMI)-1640 medium containing $10 \% \mathrm{FBS}$. All the cells were maintained at $37^{\circ} \mathrm{C}$ in a humidified incubator containing $5 \% \mathrm{CO}_{2}$.

\section{Microarrays and computational analysis}

MiR-200c-overexpressing MDA-MB-231 cells and control group MDA-MB-231 cells were selected for microarray analysis. Briefly, total cellular RNA was extracted using the TRIzol Reagent (Invitrogen, Carlsbad, CA, USA) and purified using a RNeasy Mini Kit (Qiagen, Valencia, CA, USA). Then, complementary DNA was synthesized and labeled before microarray hybridization (Arraystar, Rockville, MD, USA). The slides were washed and then scanned using an Agilent DNA Microarray Scanner (Agilent, Santa Clara, CA, USA). Data were extracted using Agilent Feature Extraction software and further analyzed using Agilent GeneSpring GX, version 12.1, software. Volcano plot filtering (fold change $>2.0$; $P<0.05)$ between miR-200c-overexpressing MDA-MB231 cells and control MDA-MB-231 cells was performed to identify lncRNAs and mRNAs showing significantly different expression. Hierarchical clustering was performed using Cluster Treeview software (Stanford, CA, USA). The microarray data have been deposited in the National Center for Biotechnology Information (NCBI) Gene Expression Omnibus (GEO) under accession number GSE119090.

\section{Lentiviral construction and transduction}

The miR-200c-3p lentiviral expression vectors were constructed by Genepharma (Shanghai, China). Genechem (Shanghai, China) constructed the lentiviral vectors expressing full-length human LINC02582. To generate clones stably overexpressing miR-200c, MDA-MB-231 and BT549 cells were infected with a lentiviral vectors expressing miR-200c-3p or an empty lentiviral vector control. To generate clones stably overexpressing LINC02582, MCF-7 and BT474 cells were infected with a lentiviral vector expressing LINCO2582 or an empty lentiviral vector control.
The U6-sh-LINC02582-CMV-GFP lentiviral vectors were constructed by Genechem (Shanghai, China) and were used to knock down the LINC02582 expression. The negative control lentiviral vector containing nonsilencing short hairpin RNA (shRNA) was used. To knock down LINC02582, MDA-MB-231 cells were infected with either of the lentiviral vectors encoding specific short hairpin RNA sequences or the negative control vector. Stable clones were selected for 2 weeks using puromycin (SigmaAldrich), and miR-200c or LINC02582 expression were detected using quantitative real-time PCR (qRT-PCR). The shRNA sequences are described in Supplementary Table 5.

\section{Plasmid construction and transfection}

The USP7, CHK1, and Ubiquitin expression plasmids were obtained from FulenGen (Guangzhou, China). For the transient transfection, cells were seeded on a six-well culture plate overnight and transfected with the plasmids or vector control by Lipofectamine 3000 (Invitrogen) according to the manufacturer's instructions.

\section{Oligonucleotides, siRNAs transfection}

The miR-200c mimic, inhibitors, USP7 small interfering RNA (siRNA), LINCO2582 siRNA, and CHK1 siRNA were purchased from RiBoBio (Guangzhou, China). Cells were seeded on the six-well culture plate and reached $50 \%$ confluence on the second day. Transfection was performed using Lipofectamine 3000 reagent (Invitrogen) according to the manufacture's protocol. After $48 \mathrm{~h}$ of transfection, the cells were used for functional assays. The sequences of siRNA are listed in Supplementary Table 5.

\section{Quantitative real-time PCR}

Total RNA was extracted from breast cancer tissues or cells using the TRIzol Reagent (Invitrogen), following the manufacturer's instructions. qRT-PCR was performed using the SYBR Green PCR kit from Takara Biotechnology (Takara, Dalian, China). For miRNA quantification, U6 was used as an internal control. GAPDH was employed as an endogenous control for quantification of lncRNA and the mRNA levels of other genes. Primer sequences are listed in Supplementary Table 6.

\section{$5^{\prime}$ and $3^{\prime}$ rapid amplification of cDNA ends}

Total RNA was extract from MDA-MB-231 cells as described about. Subsequently, 5'-rapid amplification of complementary DNA ends (RACE) was performed using a 5'-Full RACE Kit with TAP (Takara); 3'-RACE was performed using a 3'-Full RACE Core Set with PrimeScript RTase Kit (Takara), according to the manufacturer's instructions. 


\section{Luciferase assay}

To construct plasmids used in dual-luciferase reporter assays, wild-type LINC02582 cDNA (containing miR200c-binding site) and a mutant LINC02582 sequence (mutant in miR-200c-binding site) were cloned into pLUC-REPORT Vector (Promega, Madison, WI). Luciferase activities were assessed using a Luciferase Assay Kit (Promega), according to the manufacturer's instructions. Briefly, MDA-MB-231 cells were co-transfected with a luciferase reporter vector (either pLUC-WT-LINC02582 or pLUC-MUT-LINC02582) and mimic of miR-200c or negative control miRNA. The cells harvested and lysed for luciferase assays at $48 \mathrm{~h}$ after transfection. Firefly luciferase activity was used for normalization.

\section{Antibodies and western blotting analysis}

Primary antibodies included anti-USP7, anti-Ubiquitin, anti-CHK1, anti- $\gamma$-H2AX, anti-AGO2, anti-Flag, anti-HA, anti-Myc (Cell Signaling Technology, Beverly, MA, USA), and anti- $\beta$-actin (CWBIO, Guagnzhou, China). Cell pellets were lysed with radioimmunoprecipitation assay (RIPA) buffer (Cell Signaling Technology) containing proteinase and phosphatase inhibitor cocktails (SigmaAldrich). The resultant proteins were electrophoresed and transferred to a nitrocellulose membrane (Bio-Rad). Membranes were blocked with $5 \%$ bovine serum albumin for $1 \mathrm{~h}$ before incubation with primary antibodies overnight at $4{ }^{\circ} \mathrm{C}$, followed by incubation with horseradish peroxidase-conjugated secondary antibody and development with an ECL western blotting substrate (Pierce, Rockford, IL, USA).

\section{Immunofluorescence assay}

Immunofluorescence was performed by counting the $\gamma$ $\mathrm{H} 2 \mathrm{AX}$ foci at $24 \mathrm{~h}$ after irradiation. Breast cancer cells were seeded in 24-well culture plates and exposed to $6 \mathrm{~Gy}$ of irradiation. After $24 \mathrm{~h}$, the cells were fixed in $4 \%$ paraformaldehyde and permeabilized in $0.1 \%$ Triton X-100 (Sigma). The cells were then blocked in $1 \%$ goat serum and incubated with primary anti- $\gamma-\mathrm{H} 2 \mathrm{AX}$ antibodies (Cell Signaling Technology). Subsequently, the primary antibodies were washed off and the cells were incubated with secondary antibodies conjugated to fluorescein isothiocyanate. Finally, the cells were then incubated with 2(4-amidinophenyl)-1H-indole-6-carboxamidine to stain the nuclei. $\gamma-\mathrm{H} 2 \mathrm{AX}$ foci were visualized under a fluorescence microscope (Olympus BX51, Tokyo, Japan). The $\gamma-$ H2AX foci were counted for at least 50 cells in each group.

\section{Clonogenic survival assay}

Equal numbers of cells were seeded in six-well culture plates (pretreated with siRNAs or oligonucleotides, or plasmid transfected for $48 \mathrm{~h}$ ) in triplicate and exposed to the indicated doses of irradiation using 6-MV X-rays from linear accelerators (Varian2300EX; Varian, Palo Alto, CA, USA) at a dose rate of $5 \mathrm{~Gy} / \mathrm{min}$. After incubation at $37^{\circ} \mathrm{C}$ for $14-21$ days, the plates were fixed with $100 \%$ methanol and then stained with $1 \%$ crystal violet. Colonies containing $>50$ cells were counted by microscopic inspection. The surviving fraction (SF) was calculated. A multitarget single-hit model was fitted to the data to generate survival curves using the following formula: $\mathrm{SF}=1-\left(1-e^{-D / D 0}\right)^{N}$.

\section{Tumor radiosensitivity study}

All the animal experiments were carried out in strict accordance with the principles and procedures approved by the Committee on the Ethics of Animal Experiments of Southern Medical University (Guangzhou, China). Suspensions of $1 \times 10^{6} / 0.2 \mathrm{ml}$ LINC02582-silencing or control MDA-MB-231 cells were inoculated subcutaneously into the right hindlimbs of 4-week-old female BALB/c$\mathrm{nu} / \mathrm{nu}$ nude mice. Mice were randomly assigned to no irradiation or irradiation groups ( $n=4$ mice per group). Irradiation treatment was initiated when tumors grew to approximately $150 \mathrm{~mm}^{3}$. Mice in the irradiation groups were irradiated with 2 Gy every other day for five treatments. Tumor sizes were calculated every 2 days using the formula: (length $\times$ width $\left.^{2}\right) / 2$.

\section{RNA pull-down}

RNA pull-down and deletion mapping were performed as previously described ${ }^{24}$. Briefly, biotinylated LINCO2582 or antisense RNA were in vitro transcribed with the Biotin RNA Labeling Mix (Roche Diagnostics, Indianapolis, IN, USA) and T7 RNA polymerase (Roche Diagnostics), treated with RNase-free DNase I (Roche Diagnostics), and purified with the RNeasy Mini Kit (Qiagen). One milligram of protein from MDA-MB-231 cell extracts was then mixed with 40 pmol of biotinylated RNA. Incubated with streptavidin agarose beads (Invitrogen) at room temperature and washed. The associated proteins were resolved by sodium dodecyl sulfate-polyacrylamide gel electrophoresis (SDS-PAGE), and then silver-stained. Specific bands were excised and analyzed using mass spectrometry.

\section{RNA immunoprecipitation}

RIP experiments were carried out according to the manufacturer's protocol of the Magna RIP RNA-Binding Protein Immunoprecipitation Kit (Millipore, Bedford, MA). Briefly, cells were lysed with RIPA buffer (Cell Signaling Technology) containing proteinase and phosphatase inhibitor cocktails (Sigma-Aldrich). Magnetic beads (Invitrogen) were pre-incubated with primary antibodies or anti-rabbit IgG (Cell Signaling Technology) for $30 \mathrm{~min}$, and lysates were immunoprecipitated with 
beads, rotated overnight at $4{ }^{\circ} \mathrm{C}$. RNA was purified from RNA-protein complexes bounded to the beads and then was analyzed by qRT-PCR.

\section{In vivo ubiquitination assay}

For the in vivo ubiquitination assay, the $293 \mathrm{~T}$ cells were co-transfected with HA-tagged Ubiquitin, Flag-tagged CHK1, Myc-tagged USP7, LINC02582 expression plasmids, and exposed to $10 \mu \mathrm{M}$ proteasome inhibitor MG132 (Selleck, Shanghai, China) for $16 \mathrm{~h}$. The cells were lysed with RIPA buffer (Cell Signaling Technology) containing proteinase and phosphatase inhibitor cocktails (SigmaAldrich). After centrifugation 12,000 r.p.m. for $15 \mathrm{~min}$, extracts were incubated with Protein G-Plus Agarose beads (Santa Cruz Biotechnology), anti-Flag antibody (Cell Signaling Technology) was added to whole-cell lysate, and rotated overnight at $4{ }^{\circ} \mathrm{C}$. Then, the extracts were washed with lysis buffer for four times; $40 \mu \mathrm{l}$ of Protein G-Plus Agarose beads was added for another $2 \mathrm{~h}$. The agarose beads were washed and then subjected to western blotting analysis using the indicated antibodies.

\section{Immunohistochemical staining}

Immunohistochemistry (IHC) was performed on 4- $\mu \mathrm{m}$ thick sections prepared using tissue blocks embedded in paraffin. The tissue sections were fixed using $4 \%$ formaldehyde overnight and then embedded in paraffin. After deparaffinization and hydration, the sections were pretreated with sodium citrate buffer in a microwave for antigen retrieval and blocked using normal goat serum. The sections were then stained using rabbit anti-CHK1 antibodies (Cell Signaling Technology) overnight at $4{ }^{\circ} \mathrm{C}$, and then incubated in biotinylated goat anti-rabbit IgG secondary antibodies for $1 \mathrm{~h}$. Finally, the sections were stained with an avidin-biotin peroxidase complex (GeneTex, Irvine, CA, USA). Two independent pathologists performed the section scoring. The score standard for the staining extent was 1 (1-25\%), 2 (26-50\%), 3 (51-75\%) and 4 (76-100\%); and 0 (negative), 1 (weak), 2 (medium) and 3 (strong) for the staining intensity. The total scores included the extent and intensity scores ranged from 1 to 7. Total scores of $\geq 4$ defined as the high-expression group.

\section{In situ hybridization}

In situ hybridization (ISH) was used to detect miR-200c and LINC02582 in clinical breast cancer specimens, and was performed as previously described ${ }^{11}$. Briefly, the sections were deparaffinized using xylene, rehydrated in serial dilutions of ethanol, and treated with $0.2 \mathrm{~N} \mathrm{HCl}$ for $5 \mathrm{~min}$. The sections were incubated in proteinase $\mathrm{K}$ $(40 \mu \mathrm{g} / \mathrm{ml}$; Promega) for $20 \mathrm{~min}$ after washing three times. The sections were then washed in phosphate-buffered saline containing $0.2 \%$ glycine, and fixed using $4 \%$ paraformaldehyde for $10 \mathrm{~min}$. The sections were reconstituted using a hybridization solution and incubated at $56^{\circ} \mathrm{C}$ overnight with a digoxigenin-labeled locked nucleic acid (LNA)-miR-200c probe or digoxigenin-labeled LNALINC02582 probe (Exiqon, Vedbaek, Denmark). The sections were washed twice with $5 \times$ saline-sodium citrate (SSC), buffer for 20 min each time at room temperature, followed by three washes with $2 \times \mathrm{SSC}$ for $20 \mathrm{~min}$ at $50^{\circ} \mathrm{C}$. Finally, the sections were blocked with $5 \%$ normal goat serum for $1 \mathrm{~h}$ at room temperature before incubation with an anti-digoxigenin alkaline phosphatase conjugate (Roche, Stockholm, Sweden) overnight at $4{ }^{\circ} \mathrm{C}$. After staining with 5-bromo-4chloro-3indolyl phosphate (BCIP)/nitro-blue tetrazolium chloride (NBT) buffer for $10 \mathrm{~min}$, colorimetric signals were obtained by incubating the sections in BCIP/NBT buffer in the dark for $4 \mathrm{~h}$ at room temperature. Nuclear fast red was used as the counterstain. Digoxigenin-labeled LNA-scrambled miRNA or digoxigenin-labeled LNA-scrambled lncRNA probes were used as negative controls. Two independent pathologists scored the sections. The score standard for the staining extent was 1 (1-25\%), 2 (26-50\%), 3 (51-75\%) and 4 (76-100\%); and 0 (negative), 1 (weak), 2 (medium) and 3 (strong) for the staining intensity. The total scores included the extent and intensity scores ranged from 1 to 7 . Total scores of $\geq 4$ defined as the highexpression group.

\section{Statistical analysis}

All data are expressed as the mean \pm SD and statistical analysis of the data was performed using Student's $t$-test or analysis of variance (ANOVA). Spearman rank correlation was used to analyze correlations between miR-200c and LINC02582. Correlation between CHK1 and miR200c or LINCO2582 was analyzed using $X^{2}$ test. Relapsefree survival curves were plotted using the Kaplan-Meier method and compared using the log-rank test. All analyses were completed using SPSS 19.0 software (IBM Corp, Armonk, NY, USA) and $p$ values $<0.05$ were considered statistically significant.

\section{Acknowledgements \\ This work was supported by the National Natural Science Foundation of China (No. 81572964, No. 81773354, No. 81502646, No. 81872195, No. 81803170).}

\section{Author details \\ 'Department of Radiation Oncology, Nanfang Hospital, Southern Medical University, Guangzhou, Guangdong Province, People's Republic of China. ${ }^{2}$ Department of Radiation Oncology, Affiliated Cancer Hospital \& Institute of Guangzhou Medical University, Guangzhou, Guangdong Province, People's Republic of China. ${ }^{3}$ Department of Radiation Oncology, Zhejiang Cancer Hospital, Hangzhou, Zhejiang Province, People's Republic of China. ${ }^{4}$ Department of Radiation Oncology, Zhujiang Hospital, Southern Medical University, Guangzhou, Guangdong Province, People's Republic of China}

\section{Conflict of interest}

The authors declare that they have no conflict of interest. 


\section{Publisher's note}

Springer Nature remains neutral with regard to jurisdictional claims in published maps and institutional affiliations.

Supplementary Information accompanies this paper at (https://doi.org/ 10.1038/s41419-019-1996-0).

Received: 29 June 2019 Revised: 14 September 2019 Accepted: 17 September 2019

Published online: 10 October 2019

\section{References}

1. Darby, S. et al. Effect of radiotherapy after breast-conserving surgery on 10year recurrence and 15-year breast cancer death: meta-analysis of individual patient data for 10,801 women in 17 randomised trials. Lancet 378, 1707-1716 (2011).

2. Jagsi, R. Progress and controversies: radiation therapy for invasive breast cancer. CA Cancer J. Clin. 64, 135-152 (2014).

3. Jameel, J. K., Rao, V. S., Cawkwell, L. \& Drew, P. J. Radioresistance in carcinoma of the breast. Breast 13, 452-460 (2004).

4. Bracken, C. P., Scott, H. S. \& Goodall, G. J. A network-biology perspective of microRNA function and dysfunction in cancer. Nat. Rev. Genet. 17, 719-732 (2016).

5. Gebert, L. \& MacRae, I. J. Regulation of microRNA function in animals. Nat. Rev. Mol. Cell Biol. 20, 21-37 (2019).

6. Cha, Y. et al. Metabolic control of primed human pluripotent stem cell fate and function by the miR-200c-SIRT2 axis. Nat. Cell Biol. 19, 445-456 (2017).

7. Title, A. C. et al. Genetic dissection of the miR-200-Zeb1 axis reveals its importance in tumor differentiation and invasion. Nat. Commun. 9, 4671 (2018).

8. Fukuda, K. et al. Epithelial-to-mesenchymal transition is a mechanism of ALK inhibitor resistance in lung cancer independent of ALK mutation status. Cancer Res. 79, 1658-1670 (2019).

9. Lin, J. et al. miR-200c enhances radiosensitivity of human breast cancer cells. J. Cell Biochem. 114, 606-615 (2013).

10. Koo, T. et al. MicroRNA-200C increases radiosensitivity of human cancer cells with activated EGFR-associated signaling. Oncotarget 8, 65457-65468 (2017).

11. Sun, Q. et al. MiR-200c inhibits autophagy and enhances radiosensitivity in breast cancer cells by targeting UBQLN1. Int J. Cancer 136, 1003-1012 (2015).

12. Cui, F. B. et al. Enhancement of radiotherapy efficacy by miR-200c-loaded gelatinase-stimuli PEG-Pep-PCL nanoparticles in gastric cancer cells. Int J. Nanomed. 9, 2345-2358 (2014).

13. Cortez, M. A. et al. Therapeutic delivery of miR-200c enhances radiosensitivity in lung cancer. Mol. Ther. 22, 1494-1503 (2014).

14. Djebali, S. et al. Landscape of transcription in human cells. Nature $\mathbf{4 8 9}$, 101-108 (2012).

15. Huarte, M. The emerging role of IncRNAs in cancer. Nat. Med. 21, 1253-1261 (2015).

16. Schmitt, A. M. \& Chang, H. Y. Long noncoding RNAs in cancer pathways. Cancer Cell 29, 452-463 (2016).

17. Gupta, R. A. et al. Long non-coding RNA HOTAIR reprograms chromatin state to promote cancer metastasis. Nature 464, 1071-1076 (2010).
18. Liu, B. et al. A cytoplasmic NF-kappaB interacting long noncoding RNA blocks IkappaB phosphorylation and suppresses breast cancer metastasis. Cancer Cell. 27, 370-381 (2015).

19. Wang, Z. et al. IncRNA epigenetic landscape analysis identifies EPIC1 as an oncogenic IncRNA that interacts with MYC and promotes cell-cycle progression in cancer. Cancer Cell 33, 706-720.e9 (2018).

20. Chen, X., Liang, H., Zhang, C. Y. \& Zen, K. miRNA regulates noncoding RNA: a noncanonical function model. Trends Biochem. Sci. 37, 457-459 (2012).

21. Liu, Q. et al. LncRNA loc285194 is a p53-regulated tumor suppressor. Nucleic Acids Res. 41, 4976-4987 (2013).

22. Ma, M. Z. et al. Long noncoding RNA GCASPC, a target of miR-17-3p, negatively regulates pyruvate carboxylase-dependent cell proliferation in gallbladder cancer. Cancer Res. 76, 5361-5371 (2016).

23. Yang, $\mathrm{H}$. et al. Long noncoding RNA MIR31HG exhibits oncogenic property in pancreatic ductal adenocarcinoma and is negatively regulated by miR-193b. Oncogene 35, 3647-3657 (2016).

24. Tsai, M. C. et al. Long noncoding RNA as modular scaffold of histone modification complexes. Science 329, 689-693 (2010).

25. Cochrane, D. R., Spoelstra, N. S., Howe, E. N., Nordeen, S. K. \& Richer, J. K MicroRNA-200C mitigates invasiveness and restores sensitivity to microtubuletargeting chemotherapeutic agents. Mol. Cancer Ther. 8, 1055-1066 (2009).

26. Lin, C. \& Yang, L. Long noncoding RNA in cancer: wiring signaling circuitry. Trends Cell Biol. 28, 287-301 (2018).

27. Carlevaro-Fita, J. \& Johnson, R. Global positioning system: understanding long noncoding RNAs through subcellular localization. Mol. Cell. 73, 869-883 (2019).

28. Li, M. et al. Deubiquitination of p53 by HAUSP is an important pathway for p53 stabilization. Nature 416, 648-653 (2002).

29. Qing, P., Han, L., Bin, L., Yan, L. \& Ping, W. X. USP7 regulates the stability and function of HLTF through deubiquitination. J. Cell Biochem. 112, 3856-3862 (2011).

30. Colleran, A. et al. Deubiquitination of NF-kappaB by Ubiquitin-Specific Protease-7 promotes transcription. Proc. Natl. Acad. Sci. USA 110, 618-623 (2013).

31. Zhang, P. et al. ATM-mediated stabilization of ZEB1 promotes DNA damage response and radioresistance through CHK1. Nat. Cell Biol. 16, 864-875 (2014).

32. Sorensen, C. S. et al. The cell-cycle checkpoint kinase Chk1 is required for mammalian homologous recombination repair. Nat. Cell Biol. 7, 195-201 (2005).

33. Peng, W. X., Koirala, P. \& Mo, Y. Y. LncRNA-mediated regulation of cell signaling in cancer. Oncogene 36, 5661-5667 (2017).

34. Bhan, A., Soleimani, M. \& Mandal, S. S. Long noncoding RNA and cancer: a new paradigm. Cancer Res. 77, 3965-3981 (2017).

35. Marchese, F. P., Raimondi, I. \& Huarte, M. The multidimensional mechanisms of long noncoding RNA function. Genome Biol. 18, 206 (2017).

36. Smits, V. A. \& Gillespie, D. A. DNA damage control: regulation and functions of checkpoint kinase 1. FEBS J. 282, 3681-3692 (2015).

37. Alonso-de, V. I., Martin, Y. \& Smits, V. A. USP7 controls Chk1 protein stability by direct deubiquitination. Cell Cycle 13, 3921-3926 (2014).

38. Gonzalez, B. M. \& Gottifredi, V. The fork and the kinase: a DNA replication tale from a CHK1 perspective. Mutat. Res. Rev. Mutat. Res. 763, 168-180 (2015).

39. Verlinden, L. et al. The E2F-regulated gene Chk1 is highly expressed in triplenegative estrogen receptor/progesterone receptor/HER-2 breast carcinomas. Cancer Res. 67, 6574-6581 (2007). 